\title{
Connectivity modelling and network analysis of sea lice infection in Loch Fyne, west coast of Scotland
}

\author{
Thomas Adams ${ }^{1, *}$, Kenny Black ${ }^{1}$, Craig MacIntyre ${ }^{2}$, Iain MacIntyre ${ }^{3}$, Rebecca Dean ${ }^{4}$ \\ ${ }^{1}$ SAMS, Scottish Marine Institute, Dunbeg, Oban, Argyll PA37 1QA, UK \\ ${ }^{2}$ Argyll Fisheries Trust, Cherry Park, Inveraray, Argyll PA32 8XE, UK \\ ${ }^{3}$ The Scottish Salmon Company, Arkinglas Estate, Cairndow, Argyll PA26 8BH, UK \\ ${ }^{4}$ The Scottish Salmon Company, Mid-Strome, Lochcarron, Ross-Shire IV54 8YH, UK
}

\begin{abstract}
Sea lice are a persistent threat in many areas where salmon farming is practised. In common with the management of disease, infection levels are typically controlled by operating sites within distinct geographical areas, allowing for coordinated treatment and fallow cycles. However, the hydrodynamic connectivity and consequent transmission of lice larvae between sites is often not well understood, which limits our ability to optimise the spatial distribution of farms to minimise infection. We used a multistage modelling approach to investigate the transmission of sea lice larvae between salmon aquaculture sites in Loch Fyne, Scotland. A finite element hydrodynamic model was forced using meteorological data collected over the study period. Output from this model was used to drive a particle-tracking model. The latter model implemented the development and mortality of larvae to estimate the probability of successful larval dispersal between sites. In turn, these dispersal probabilities were used to define a network describing the sea lice metapopulation (its habitat defined by the aquaculture sites). Methods from graph theory allow the identification of those sites in the network that are likely to be key for the control of sea lice in the loch population as a whole. Model outputs were compared with data from a campaign of plankton tows and with lice abundance data from aquaculture sites. The general pattern of abundance was reasonably well replicated, albeit with some notable discrepancies. These differences are worth investigating further, as they may be suggestive of sources of infection by wild fish or of inadequacies in the model.
\end{abstract}

KEY WORDS: Sea lice · Connectivity · Biophysical model $\cdot$ Larval dispersal $\cdot$ Population dynamics · Lepeophtheirus salmonis

\section{INTRODUCTION}

Seawater Atlantic salmon Salmo salar L. aquaculture sites are best operated within distinct management areas, whether this is for the purposes of disease management (Werkman et al. 2011), the control of sea lice or other operational factors (Bjørn et al. 2011, Code of Good Practice Management Group 2011). The spatial arrangement of these areas is designed to allow synchronous fallowing and treatment for pathogens, thereby reducing cross-infection between farms. However, the connectivity of farms both between and within management areas is very poorly understood, as is the optimal configuration of boundaries for management areas. This poses a problem for our understanding of the exact nature of the transmission vector.

In the context of salmon farming, a particular problem is posed by 'sea lice', a type of parasitic copepod. While endemic in wild salmon populations (now and prior to the advance of intensive fish farming), it is in farmed populations that their presence becomes most obvious (Costello 2006). Here, large numbers of available host fish can lead to high parasite infection 
rates unless the farm is well managed. Impact depends on fish size and life history stage. Relatively few lice may be lethal to smolts (Heuch et al. 2005) and can lead to increased mortality rates in juvenile fish (Morton \& Routledge 2005), but large numbers can also lead to serious damage to individual adult fish (Costello 2006). The primary species affecting salmonids in Scottish waters is Lepeophtheirus salmonis (L.). Although not specific to salmonids, Caligus elongatus (L.) can also cause a lesser problem for farmed fish.

Adult female sea lice produce 2 egg strings at a time, each consisting of between 70 and 290 eggs (Stien et al. 2005). Upon hatching, eggs produce noninfective nauplii. These may be capable of short bursts of movement (Heuch \& Karlsen 1997) and may exhibit chemotaxic behaviour at short ranges (Bailey et al. 2006), but in general, the nauplii move passively in the water column and are dispersed with the current on larger scales. They develop into copepodids at a rate dependent upon water temperature, and once they have done so, become infective.

Understanding sea lice transmission between farm sites is thus a problem of understanding local hydrodynamics. Insights gained have a number of important commercial and environmental benefits. Infection rates can be reduced by effective management, but once infected, sea lice and other pathogens are dealt with either by chemical treatments (Burridge et al. 2010) or occasionally by use of cleaner fish (Costello 2006). As medicines are both expensive to administer and are ultimately released into the environment, reduction in their use is highly desirable. Also, repeated medicine use increases the risk of the development of lice strains that are drug-resistant, reducing drug efficacy (Lees et al. 2008). Gaining an understanding of infection pathways may allow managers to optimise and reduce chemical treatments, or to control infections without resorting to their use. Sea lice transmission information may also have other benefits, such as reduced cross infection with wild salmonids, reduced risk from inter-farm infection with important notifiable diseases (e.g. infectious salmon anaemia), leading to reduced risk of catastrophic epidemics (Aldrin et al. 2011). All of these factors are likely to increase the sustainable farmed capacity, both financially and ecologically.

Connectivity modelling is becoming more widely applied in attempts to understand the population dynamics of marine organisms (e.g. North et al. 2008, Treml et al. 2008, Fox et al. 2009). While some results are specific to the geography of the study region, such studies allow identification of generalities in lar- val dispersal patterns, such as the importance of vertical migration (Fox et al. 2006, Sundelöf \& Jonsson 2011), or the role of particular sites as a source or sink for larvae (North et al. 2008, Ayata et al. 2010). A model provides a well-defined baseline against which to compare empirical observations, and appropriate models can provide testable hypotheses and give insight into specific aspects of biological or hydrodynamic processes in the real world. Several studies have already hinted at this strength (Fox et al. 2006, Knights et al. 2006).

Models similar to ours have already been implemented to investigate sea lice transmission in other areas of Scotland (Amundrud \& Murray 2009, Salama et al. 2011), Norway (Asplin et al. 2004, Askeland Johnsen 2011) and Canada (Stucchi et al. 2010). We built upon this body of work, considering the implications of viewing the network of sites as a metapopulation of sea lice. Defining a network by the larval dispersal probabilities between each site (generated by a particle-tracking model) allows the application of graph theoretic techniques to understand the role of sites in overall connectivity and to identify key targets for management.

Here we document our attempts to understand the larval dispersal of sea lice among a network of aquaculture sites within a system that, excepting occasional long dispersal events (Gilbert et al. 2010), is effectively closed to infection by larvae from farms elsewhere (the nearest sites outside the management area are near Arran, at least $25 \mathrm{~km}$ away). We used a combination of hydrodynamic and biological models, and analysed outputs using metrics from graph theory. This enables the identification of key sites for management, and an understanding of the main dispersal pathways in the loch. In doing so, we hope that insights may be gained into farm siting, improving timing and reducing intensity of treatments, as well as reducing transmission between farmed and wild fish.

\section{METHODS}

\section{Study system}

Sea lochs are fjordic arms of the sea that reach for many miles inland, and are the predominant feature of the coastal environment on the west coast of Scotland. Of these, Loch Fyne is one of the longest $(61 \mathrm{~km})$ and deepest $(185 \mathrm{~m}$; Edwards \& Sharples 1986). We defined the study region (Fig. 1) as the entirety of Loch Fyne, bounded on the south at its opening into the Clyde basin. This study region is 


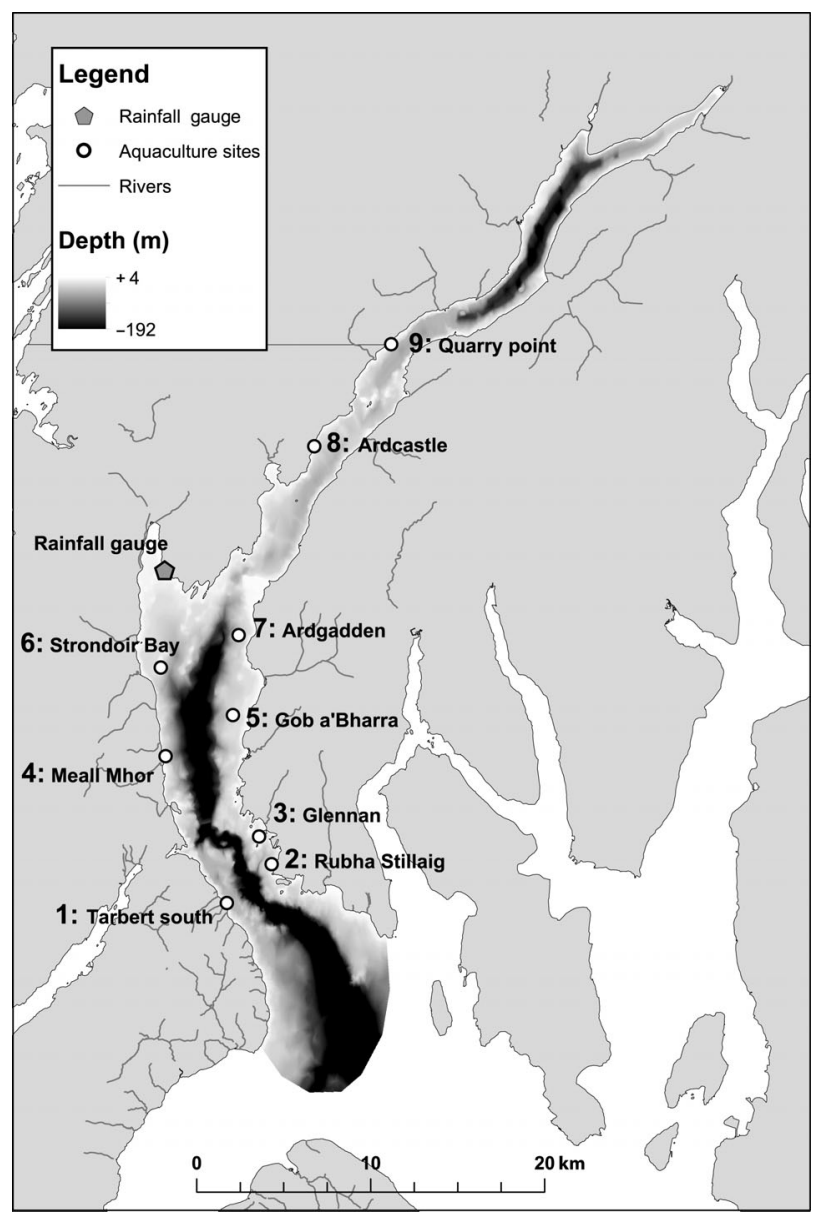

Fig. 1. Loch Fyne, on the west coast of Scotland, showing locations of aquaculture sites and the Scottish Environmental Protection Agency (SEPA) rainfall gauge at Lingerton. Anemometers were located at Sites 6 and 9. Coastline, hydrology and bathymetry data were obtained from Edina Digimap. Depth values relative to mean sea level

relatively simple in terms of tidal forcing (there is a single route for tidal waters to enter and leave), and its geographical shape means that meteorological influences are relatively simple to study and estimate. However, the modelling of sea lochs such as Loch Fyne is complicated by features such as sills (shallow sections between basins) and high levels of freshwater input, which leads to strong stratification and long timescale flushing events.

\section{Hydrodynamic model}

To study water movements in Loch Fyne, we implemented the Finite Volume Coastal Ocean Model (FVCOM). Precise details of the computations are available elsewhere (Chen et al. 2006). In brief, the model computes fluxes between elements of a predefined mesh in order to simulate the dynamics of a water body (currents, salinity and temperature), using a system of differential equations. Triangular elements allow variation in element size, which means that regions with complicated topography can be resolved with increased detail. This feature is particularly important in fjordic coastal environments, which contain features at many different spatial scales, and are impossible to represent accurately with reasonable computational cost in a regular grid model. Two mesh resolutions were implemented, with similar circulation patterns being observed in both (not shown). Principal hydrodynamic statistics at the aquaculture sites were not improved by the fine-scale model (see the supplement at www.intres.com/articles/suppl/q003p051_supp.pdf), so for computational efficiency, the coarser of the 2 meshes was used here (consisting of 943 elements, with 10 vertical layers concentrated near the surface; see Fig. S1 in the supplement).

FVCOM was used in a baroclinic configuration. We included wet/dry treatment of individual mesh elements and various types of external input forcing. Ten major freshwater inputs (rivers) were included, with flow rate based upon a moving average of daily rainfall measured at Lingerton (Scottish Environmental Protection Agency, SEPA; see Fig. 1). Open boundary tidal forcing used 6 principal constituents obtained from Zijl (2009), in addition to temperature and salinity nudging (ensuring that any input at the boundary was 'ocean' water). A 'sponge' (mixing) region at the open boundary prevents discontinuity when freshwater reaches the boundary by allowing it to mix properly with the saline water. The timestep for stable model integration has an upper bound, limited by the maximum possible wave speed in the smallest horizontal mesh element and deepest section of the bathymetry. A value of $0.621 \mathrm{~s}$ was used, which satisfies this condition.

Realistic wind data are instrumental in the accurate modelling of dispersal patterns (Amundrud \& Murray 2009), and to this end, we installed 2 weather stations, at Strondoir Bay and Quarry Point aquaculture sites, from March 2011 to February 2012. The collected weather station timeseries displayed correlation throughout the year. Small-scale variation in wind patterns may play a role in particular areas of the loch, but this was not accounted for here. In the model, wind forcing was assumed to vary in time (average hourly values were used) but to be homogeneous in space (as the loch is largely oriented in 1 direction). 
Hydrodynamic simulations over 1 mo were performed for each of the 12 calendar months within the data collection period. Further 4 mo simulations were also performed, although we used these for salinity validation runs rather than for particle-tracking simulations. The hydrodynamic model was validated against: (1) historic current records made at the aquaculture sites; (2) salinity data from conductivity, temperature, density (CTD) devices installed for the duration of the project; and (3) salinity data collected on a transect by Gillibrand (2002).

\section{Particle-tracking (biological) model}

\section{Description}

Following Amundrud \& Murray (2009), we defined a biological model that includes movement, maturation and mortality. Movement of individual simulated lice larvae is determined by the currents computed in the hydrodynamic model. Due to a lack of evidence suggesting definite vertical migration patterns, we assumed that lice larvae remain in the upper reaches of the water column (Murray \& Gillibrand 2006), and approximated their velocity by an inverse-distance weighted average of velocity in the upper layer of the 5 nearest element centroids. Lice inhabiting different depth layers are considered in the 'Results'. The distance travelled due to current in the model is $\Delta r_{\text {current }}=$ $\Delta t \times v$, where $v$ is the current velocity at the larva's location. As per Murray \& Gillibrand (2006), additional movement due to diffusion is incorporated by adding a term $\Delta r_{\text {diffusion }} \sim \sqrt{6 D_{h} \Delta t} \times U(-1,1)$, with $D_{h}=$ 0.1 (Turrell 1990). Different values were tested but did not have a marked effect within our study region. Note that $r$ and $V$ are vector quantities (i.e. $r=[x, y]$ and $v=[\mathrm{d} x / \mathrm{d} t, \mathrm{~d} y / \mathrm{d} t])$. The total distance travelled in 1 timestep is thus $\Delta r=\Delta r_{\text {current }}+\Delta r_{\text {diffusion. }}$. At each timestep, each larva's location is updated as $r_{t+1}=r_{t}+$ $\Delta r$ (a simple Euler scheme). If a computed trajectory step would have taken a particle onto land, the particle remained at its existing position (Stucchi et al. 2010).

The maturation rate of sea lice larvae is dependent upon the ambient water temperature. In all simulations presented, water temperature is assumed to be $10^{\circ} \mathrm{C}$, giving a minimum age of maturity of $3.63 \mathrm{~d}$ (based on the calculation of Stien et al. 2005). The annual temperature observed at the farm sites was 8 to $14^{\circ} \mathrm{C}$, over which Stien et al. (2005) estimated larval maturation rate to be fairly insensitive. At this point, all larvae are assumed to become infective, giving a slightly more rapid development than reported by Amundrud \& Murray (2009), who assumed that individual lice mature after this time at a rate of $0.1 \mathrm{~h}^{-1}$.

Due to contradictory results obtained by studies on the determining factors of sea lice mortality, Amundrud \& Murray (2009) assumed a constant rate of mortality $\left(0.01 \mathrm{~h}^{-1}\right)$ for lice at all life stages. Although homogeneous mortality affects absolute concentration, it does not affect relative distribution of larvae. Furthermore, constant mortality can be applied to particle-tracking results 'off-line', based on the recorded arrival times at locations of interest (Treml et al. 2008). We followed this approach and calculated that the proportion of a 'packet' of larvae arriving $t$ hours after a release event that have survived until that time is $e^{-0.01 t}$. All model larvae die if they do not find a host after $14 \mathrm{~d}$.

Allowing 1 wk for hydrodynamic model 'spin-up', 1000 particles are released from each site over a $25 \mathrm{~h}$ period (to reduce the impact of tidal forcing on the results). This provides a balance between computational efficiency and smooth estimation of density; simulations using fewer particles resulted in more stochastic distribution patterns. In our model, larvae are deemed to be successful in reaching a destination site if they enter a region of $500 \mathrm{~m}$ radius about the site. Smaller radii reduced the absolute value of larval arrival densities but did not notably alter relative values of the main metrics computed in 'Network analysis' below.

\section{Scenarios}

We enacted scenarios investigating the impact of wind forcing and freshwater supply. The collection of wind data over an entire year allowed us to investigate the impact of realistic variation in wind forcing. Particle-tracking simulations using the final $3 \mathrm{wk}$ of each calendar month were performed to assess sensitivity of dispersal predictions to this variation.

The importance of increased freshwater supply to dispersal was also considered. Varying freshwater discharge within realistic ranges did not lead to notable variation in circulation patterns. In sea lochs, a stratified layer of freshwater often sits above more saline water (particularly during periods of high rainfall). The project coincided with a period of unusually high rainfall (approximately $2200 \mathrm{~mm}$ in 2011; SEPA Lingerton data), compared to an average of $1750 \mathrm{~mm}$ (Edwards \& Sharples 1986); regional averages have remained fairly stable over the last 25 yr (Met Office data not shown). It is thought that adult sea lice can- 
not survive long periods in freshwater (McLean et al. 1990), and previous studies have found lice to be absent from surface waters during periods of heavy rainfall (Costelloe et al. 1998), leading to the idea that they might migrate vertically to avoid this influx of freshwater. Particle-tracking simulations were run using identical forcing, but with particles assumed to inhabit layers 2 (mean depth $4.98 \mathrm{~m}$ ) and 3 (mean depth $8.3 \mathrm{~m}$; mean depth in layer 1 is $1.66 \mathrm{~m}$ ), to determine whether vertical migration of larvae influenced dispersal patterns.

\section{Network analysis}

Particle tracking outputs the source, destination and dispersal time of each particle that successfully disperses between sites. This can be used to define a 'metapopulation' network. The probability of a particle in the model successfully dispersing from site $i$ to site $j$ is

$$
P_{i j}=\sum_{n=1}^{N_{i}} \frac{\mathrm{e}^{-0.01 t_{i j n}}}{N_{i}}
$$

where $N_{i}$ is the number of particles released from site $i$, and $t_{i j n}$ is the time taken by the $n$th particle to travel between the sites. Summing all values $P_{i j}$ and dividing by the number of sites gives the average probability of successful dispersal. The matrix of all pairwise probabilities is not symmetric (i.e. $P_{i j} \neq P_{j i}$ ). Site influx is defined as $P_{j}=\sum_{i=1}^{n s i t e s} P_{i j}$ i.e. the relative number of arrivals at each site, assuming that all sites have identical larval output (their role as a 'sink' for larvae). To describe to extent to which a site behaves as a 'source', we define outflux as $P_{i}=\sum_{j=1}^{n s i t e s} P_{i j}$ i.e. the relative proportion of successful dispersal events starting at a site. The values of $P_{i j}$ could be weighted by source site-specific information, such as host fish biomass.

Self-infection is also considered to be of particular interest in explaining parasite burdens at aquaculture sites (Krkošek et al. 2010). The self-infection probability of a site is given by the terms on the leading diagonal of the connectivity matrix (i.e. $P_{j j}$ ). The level of self-infection relative to external infection $\left(P_{j j}=\sum_{i=1, i \neq j}^{n s i t e s} P_{i j}\right)$ was also considered; sites that have a relatively high level of self-infection are more likely to experience positive feedback through reproduction and thus higher lice levels.

Considering the most probable ('shortest') pairwise paths allows us to rank the importance of nodes to overall connectivity. The number of shortest paths between all pairs of nodes that a node lies on is its 'betweenness'. Nodes with high betweenness are important to overall connectivity and are thus obvious targets for management. There may also be nodes which, when removed, break the network into 2 or more separate networks ('cut-nodes'). Again, these are obvious targets for management, as they are likely to be important for general pathogen transmission.

\section{Observational data}

For model validation purposes, plankton samples of surface water (with a tow distance of 50 to $100 \mathrm{~m}$ ) were collected at 13 locations in Loch Fyne, once each month between March 2011 and February 2012. Dividing the number of lice larvae by tow water volume gives the larval concentration.

We also analysed lice count data obtained from salmon aquaculture sites in Loch Fyne. Data were collected by the fish farmers at each of the sites. Counts of Lepeophtheirus salmonis and Caligus elongatus were made, but the latter were not included in this analysis because there were so few of them. In general, 5 fish were sampled from each cage of the farm at each sampling event (in line with the recommendations of the Code of Good Practice Management Group 2011, and also following the suggestion of Heuch et al. 2011). The frequency of sampling varied throughout the sampling period of interest (2010 to 2011), with increased sampling frequency in the summer months, although samples were collected at least once each month throughout the production cycle. Counts were given for all of the main life history stages. Information on total fish numbers, average fish weight, temperature, total number of fish sampled and anti-parasitic medication dates was also provided.

\section{RESULTS}

\section{Hydrodynamic model}

In order to determine the accuracy of the hydrodynamic model, we compared model output with (1) historic current records from the aquaculture sites; (2) salinity data from CTD devices installed for the duration of the project; and (3) salinity data collected on a transect by Gillibrand (2002).

Acoustic Doppler current profiler records were available for $14 \mathrm{~d}$ periods at each aquaculture site (except Tarbert South, Site 1). The records are historic, and from different periods. Accompanying 
weather data were not available; we simply know that each record was made during a 'permissible' period (during which adverse weather conditions did not occur), and so we compared these data with model output for $14 \mathrm{~d}$ periods in the absence of wind forcing. Mean current speeds generally fitted well (Fig. 2a), but at most sites very low currents occur more frequently in reality than in the model results (Fig. 2b). This means that the model overestimates residual flow (current velocity vectors at a single location plotted end to end, giving a notional trajectory from that location, so called 'progressive vectors') at most sites (Fig. 2c), although the rankings of the sites are broadly correlated. This is not surprising. Aside from the short duration and unknown meteorological conditions, the spatial locations of

(a) Mean current speed $\left(\mathrm{m} \mathrm{s}^{-1}\right)$

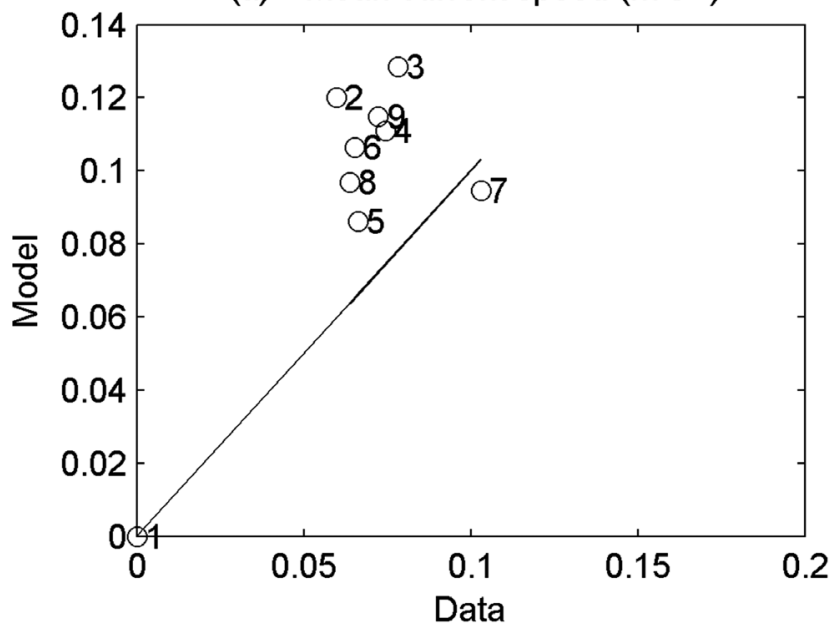

(c) Residual flow speed $\left(\mathrm{m} \mathrm{s}^{-1}\right)$

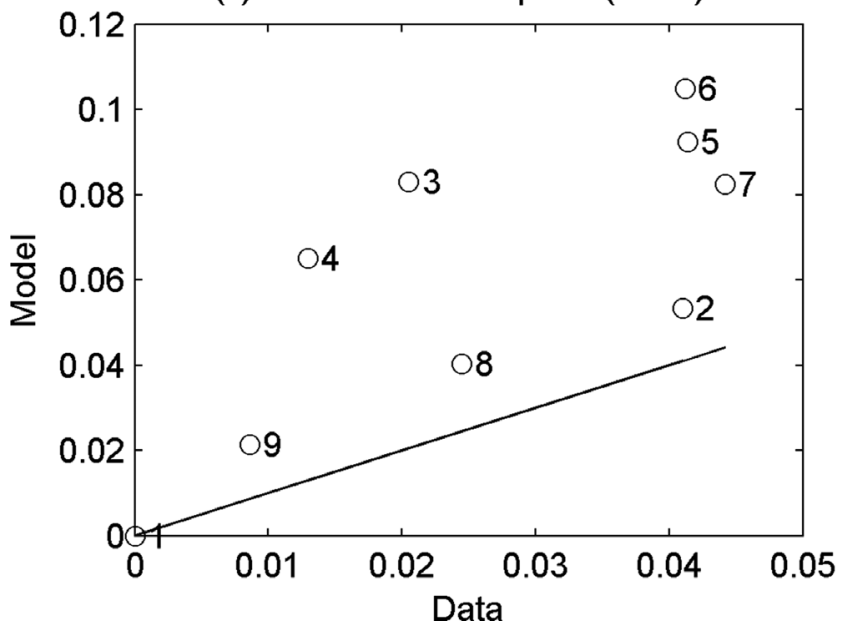

model current points may differ from those of the site current meters by up to a few hundred metres, due to uncertainty in the precise meter location, and the approximated model site location. Current meter data are also likely to display the effects of processes not represented in the model, such as lee effects and eddies created by the farms near instrument deployment sites. 'Equivalent' model points are located in open water. Residual flow direction (Fig. 2d) fitted well for some sites, but notably badly for Sites 2 and 7. At both sites, there was a discrepancy of approximately $180^{\circ}$, and a high (well matched) residual flow speed. This could be due to wind direction during the data collection, but the differences are worth keeping in mind during later consideration of connectivity.

In work to construct and validate a 2-dimensional
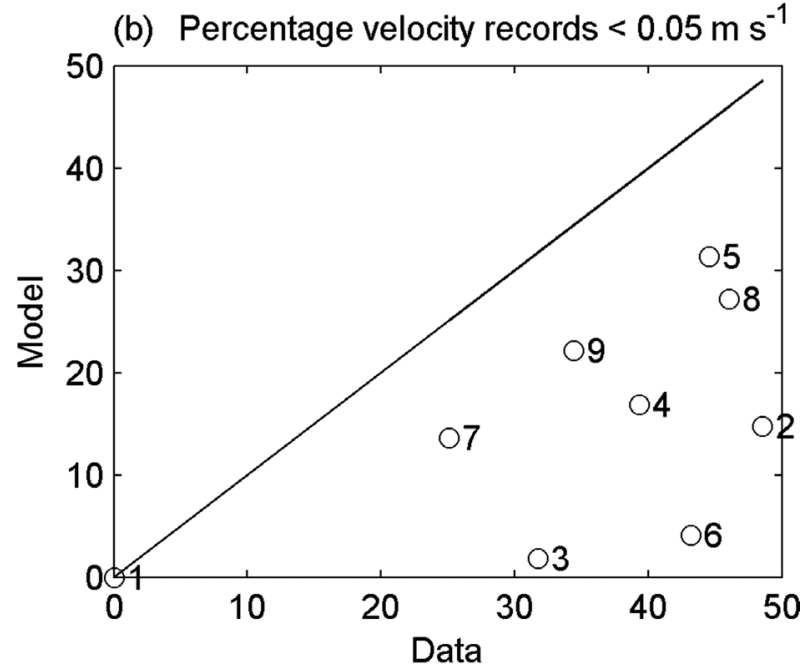

(d) Residual flow direction

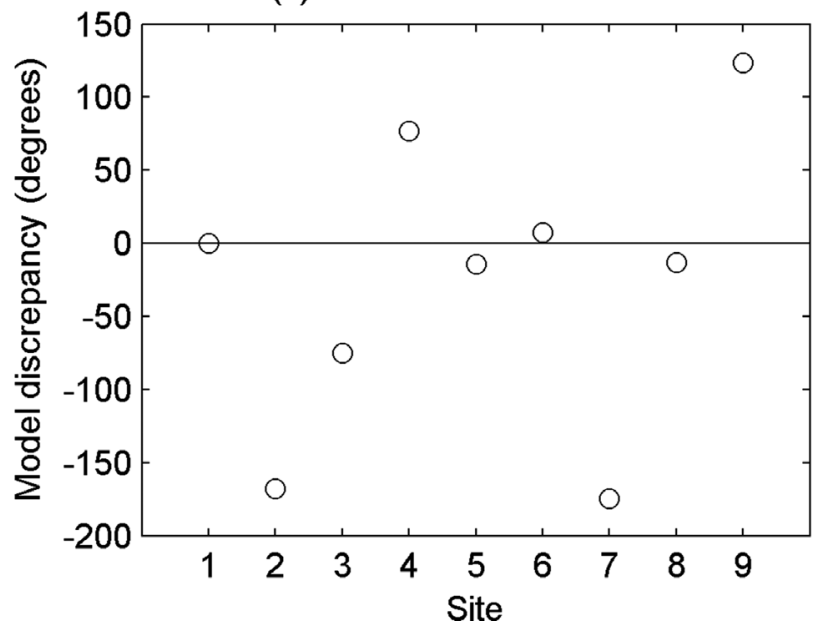

Fig. 2. Comparison of main current statistics measured at aquaculture sites (see Fig. 1) in Loch Fyne with model output in the absence of wind forcing ( $2 \mathrm{wk}$ long current record). No data were available for Site 1 . The solid line represents the ideal case where model = data. (a) Mean current speed. (b) Percentage of velocity records less than $0.05 \mathrm{~m} \mathrm{~s}^{-1}$. (c) Residual flow speed.

(d) Model discrepancy in residual flow direction 
circulation model, Gillibrand (2002) collected salinity data over the full depth of Loch Fyne at 14 locations along its longitudinal axis (on 21 November 1994). The overall salinity pattern in the loch compares well, but our model generally predicts salinity around 1 psu below Gillibrand's (2002) observed values (not shown). This result is replicated when comparing time series data recorded during the course of the project with equivalent fixed location $(x, y, z)$ time series from the model (when forced with contemporary rainfall/estimated river outflow, data not shown). Short-term circulation in the model is relatively insensitive to exact salinity (data not shown). The biological model implemented is also independent of salinity, so this discrepancy is not expected to influence the results.

\section{Dispersal patterns and sensitivity to meteorology}

\section{Wind forcing}

We found that continuous homogeneous wind forces modelled larvae (as might be expected) farther north-east in the loch than the 'no wind' case (Fig. 3). However, we found that temporally variable 'realistic' wind pushes the particles farther still along the loch. Computing the mean westerly and southerly components of the input wind data in this case gives a mean wind direction of $205.1^{\circ}$, and speed of $1.88 \mathrm{~m} \mathrm{~s}^{-1}$. This is broadly similar to the constant wind scenario, although clearly there is great variation in direction and speed over the period. It appears that this variation may assist particles in moving along the loch, despite its predominant orientation.

This is understood more clearly by considering connectivity matrices in each case. Summing the probabilities of connection over all sites, we generally found that connectivity is increased in the cases including wind. For example, the chance of successful dispersal (that is, taking account of larval duration and mortality) is $3.3 \%$ in the 'no wind' case, $4.3 \%$ in the 'March wind' case and $4.7 \%$ in the 'constant wind' case.

In addition to altering the overall connectivity of the network of aquaculture sites, changes in wind also alter the importance of the different sites in the loch predicted by the model. We ran 12 separate simulations using different monthly wind forcing. The average connectivity (and indeed connectivity in the absence of wind forcing) is limited by seaway distance, but a great level of variation is observed, with many relatively close pairs being poorly connected (Fig. 4). The average connection probabilities between each pair of sites (including 'self pairs') are shown in Table 1. (a)

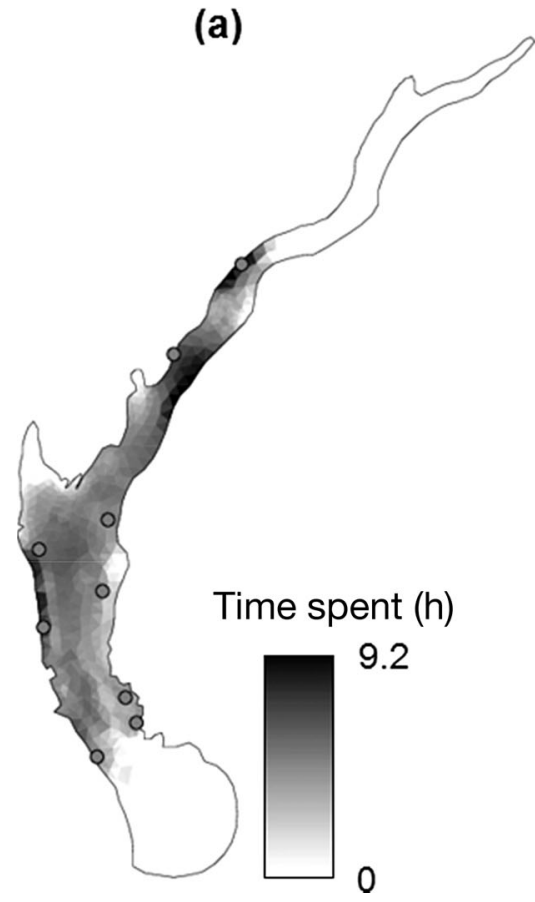

(b)

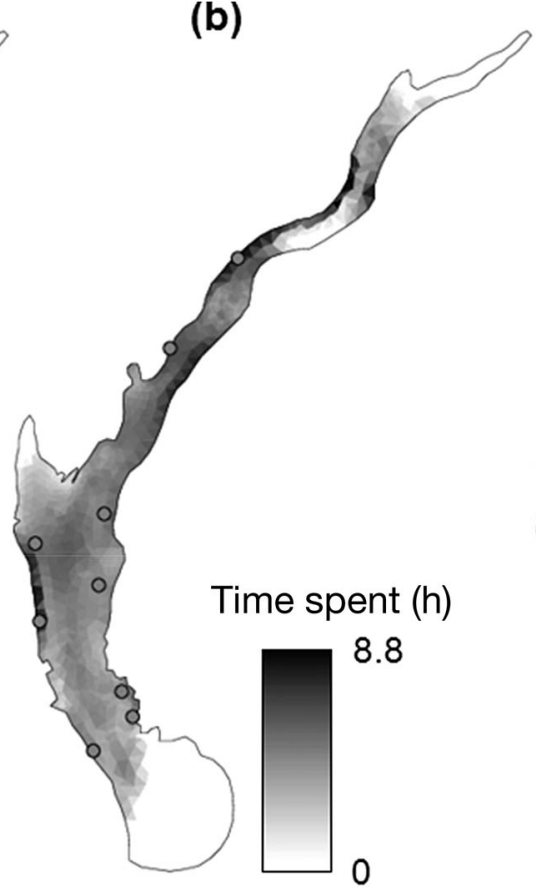

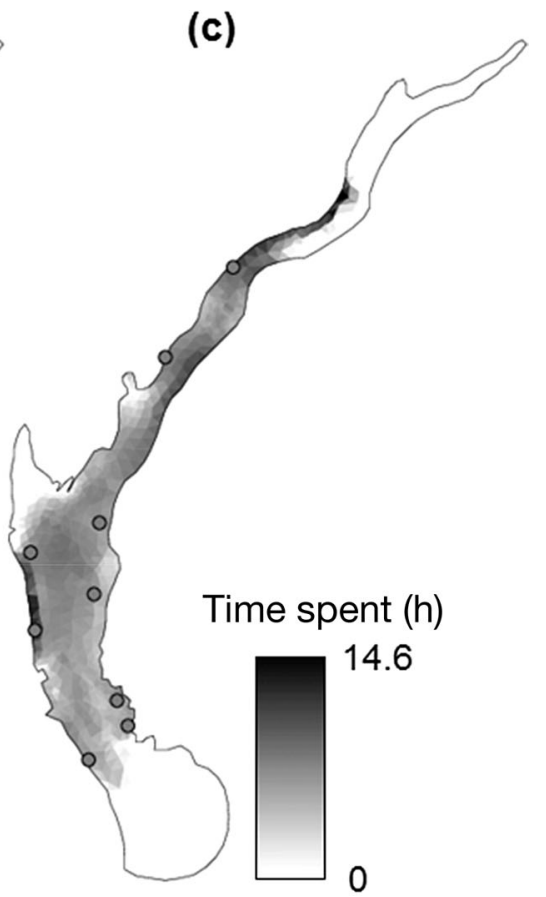

Fig. 3. Average number of hours spent in each element by each particle over the ensemble of all particle track simulations (assuming no mortality). The spatial spread of particles varies with wind forcing: (a) no wind, (b) March 2011 winds, and (c) constant $2.25 \mathrm{~m} \mathrm{~s}^{-1}$ south-westerly winds. Circles: aquaculture sites, as in Fig. 1 


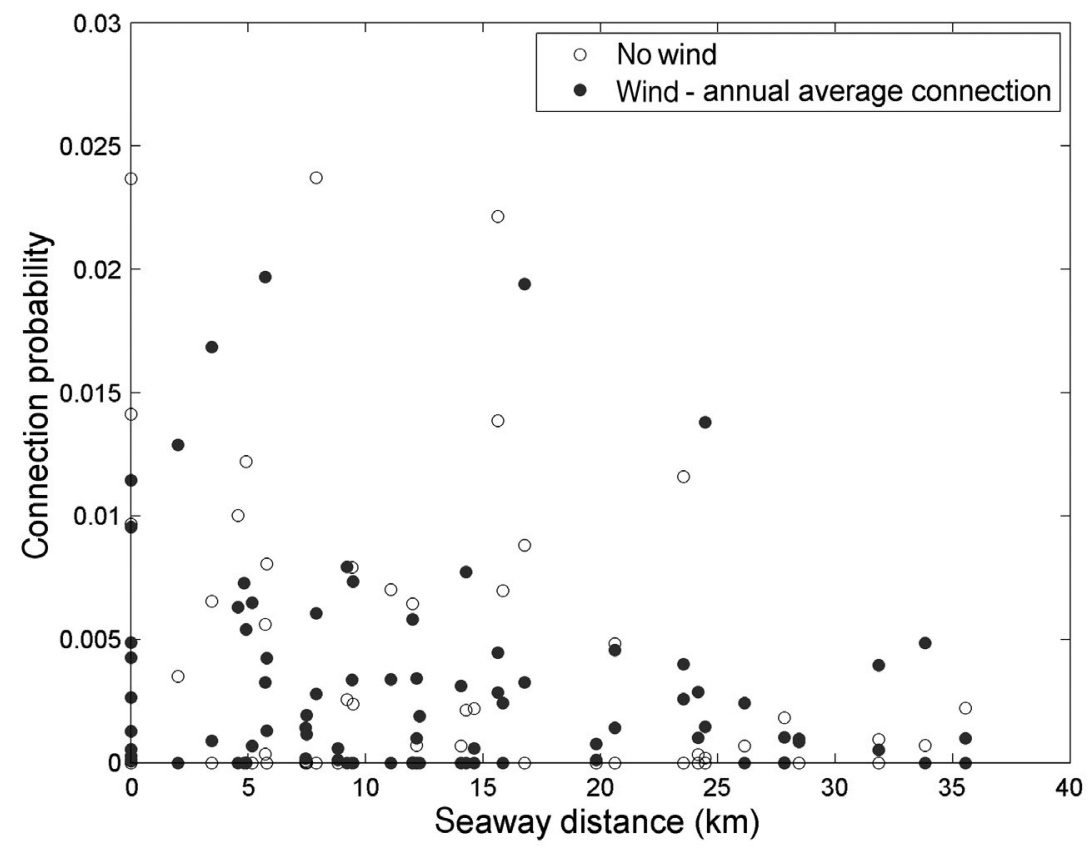

Fig. 4. Connection probabilities versus seaway distances between sites for simulations with no wind forcing (open circles), and the annual average connection between sites over 12 separate simulations with observed monthly wind forcing. Distance imposes a limit on connection strength, but very large variability is seen at all spatial scales

Fig. 5 shows the graph/connectivity metrics identified above (self-infection, influx, outflux and betweenness) for each site using the 12 different monthly wind forcing scenarios. Average self-infection rates varied between sites, but much greater variation was observed at particular sites depending on the weather conditions (e.g. Sites 3 and 8). Influx is highest at Site 9 (NE end of loch) and Site 5 (central eastern shore), but (particularly for the former) is highly dependent upon the wind forcing, having high influx early in the year and relatively low influx later on (data not shown). Sites at the south end of the loch do not see high influx of larvae under any modelled wind scenario. Sites farther south in the loch tend to have the highest outflux, but again this varies by a factor of 2 in extreme cases of wind forcing. Despite apparent correlations in outflux of the sites over the year, some sites are consistently high (Sites 4 and 6, both located on the west shore of the loch). Outflux from Site 9 is 0 in almost all scenarios. Betweenness shows large amounts of variation between months, but Sites 7 and 3 have the highest values (Site 3 being the most consistent).

Freshwater supply and vertical migration

Particles subject to water movements in hydrodynamic model layers at greater depths do not move around as readily as those near the surface. Overall, there is a $4.4 \%$ chance of successful dispersal for particles in layer 1, 4.3\% in layer 2 and $3.6 \%$ in layer 3 .

Influx at sites in the south and outflux at sites in the north of the loch are somewhat heightened at greater depths. Other sites are not notably affected, and betweenness of sites does not change over the 3 depths considered (data not shown).

\section{Model results and observational data}

Plankton tows did not yield many sea lice larvae; in fact, only 12 of 126 sampling events (each consisting

Table 1. Average inter-site connection probabilities. Source site is given by row, and destination by column. 'Self-infection' probabilities are given by the leading diagonal elements (in bold). The ratio of this term to the sum of other terms in the column (the ratio of selfinfection to external infection) is given in the bottom row

\begin{tabular}{|c|c|c|c|c|c|c|c|c|c|c|}
\hline & & 1 & 2 & 3 & 4 & $\begin{array}{c}\text { Destination } \\
5\end{array}$ & 6 & 7 & 8 & 9 \\
\hline \multirow[t]{9}{*}{ Source } & 1 & $1.28 \times 10^{-3}$ & $1.19 \times 10^{-4}$ & $9.78 \times 10^{-4}$ & $3.99 \times 10^{-3}$ & $1.38 \times 10^{-2}$ & $6.06 \times 10^{-3}$ & $3.96 \times 10^{-3}$ & $9.88 \times 10^{-4}$ & $4.85 \times 10^{-3}$ \\
\hline & 2 & $7.76 \times 10^{-4}$ & $1.08 \times 10^{-4}$ & $5.87 \times 10^{-4}$ & $5.41 \times 10^{-3}$ & $7.28 \times 10^{-3}$ & $5.82 \times 10^{-3}$ & $1.89 \times 10^{-3}$ & $2.42 \times 10^{-3}$ & $7.74 \times 10^{-3}$ \\
\hline & 3 & $8.47 \times 10^{-4}$ & $1.14 \times 10^{-4}$ & $5.51 \times 10^{-4}$ & $4.23 \times 10^{-3}$ & $6.50 \times 10^{-3}$ & $4.56 \times 10^{-3}$ & $1.42 \times 10^{-3}$ & $3.37 \times 10^{-3}$ & $7.95 \times 10^{-3}$ \\
\hline & 4 & $2.59 \times 10^{-3}$ & $1.37 \times 10^{-5}$ & $1.30 \times 10^{-3}$ & $2.64 \times 10^{-3}$ & $1.97 \times 10^{-2}$ & $4.47 \times 10^{-3}$ & $3.43 \times 10^{-3}$ & $5.93 \times 10^{-4}$ & $3.11 \times 10^{-3}$ \\
\hline & 5 & $1.47 \times 10^{-3}$ & 0 & $6.84 \times 10^{-4}$ & $3.26 \times 10^{-3}$ & $9.56 \times 10^{-3}$ & $3.25 \times 10^{-3}$ & $1.16 \times 10^{-3}$ & $3.38 \times 10^{-3}$ & $7.36 \times 10^{-3}$ \\
\hline & 6 & $2.79 \times 10^{-3}$ & $2.00 \times 10^{-5}$ & $1.42 \times 10^{-3}$ & $2.85 \times 10^{-3}$ & $1.94 \times 10^{-2}$ & $4.87 \times 10^{-3}$ & $2.88 \times 10^{-3}$ & $1.02 \times 10^{-3}$ & $2.42 \times 10^{-3}$ \\
\hline & 7 & $5.23 \times 10^{-4}$ & 0 & $1.79 \times 10^{-4}$ & $9.94 \times 10^{-4}$ & $1.94 \times 10^{-3}$ & $1.01 \times 10^{-3}$ & $2.67 \times 10^{-4}$ & $6.31 \times 10^{-3}$ & $1.29 \times 10^{-2}$ \\
\hline & 8 & 0 & 0 & 0 & 0 & 0 & $8.51 \times 10^{-6}$ & 0 & $4.27 \times 10^{-3}$ & $1.69 \times 10^{-2}$ \\
\hline & 9 & 0 & 0 & 0 & 0 & 0 & 0 & 0 & $8.94 \times 10^{-4}$ & $1.15 \times 10^{-2}$ \\
\hline \multicolumn{2}{|c|}{ Self:external } & 0.14 & 0.41 & 0.11 & 0.13 & 0.14 & 0.19 & 0.02 & 0.22 & 0.18 \\
\hline
\end{tabular}


Fig. 5. Graph metrics by site, based on connectivity matrices generated by 12 wind forcing scenarios spanning March 2011 to February 2012. (a) Probability of self-infection. (b) Influx: total probability of larvae arriving at each site, assuming equal larval output. (c) Outflux: probability of successful dispersal from each site. (d) Betweenness: number of shortest paths on which the site lies, of a possible maximum of 81 . For full details, see 'Network analysis' in 'Methods'. Solid line is the median value, box ends at $25 \%$ quartiles, and whiskers are $90 \%$ interval (a) Self-infection

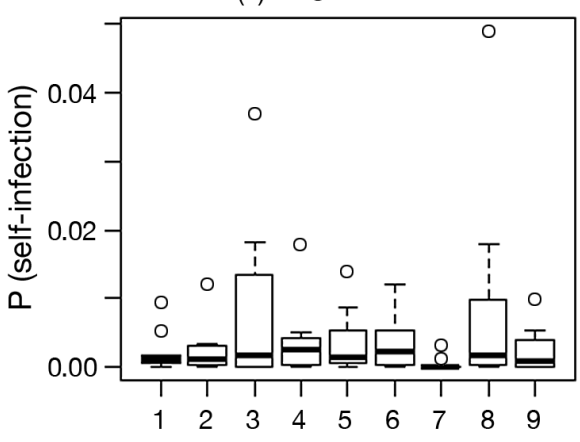

(c) Outflux

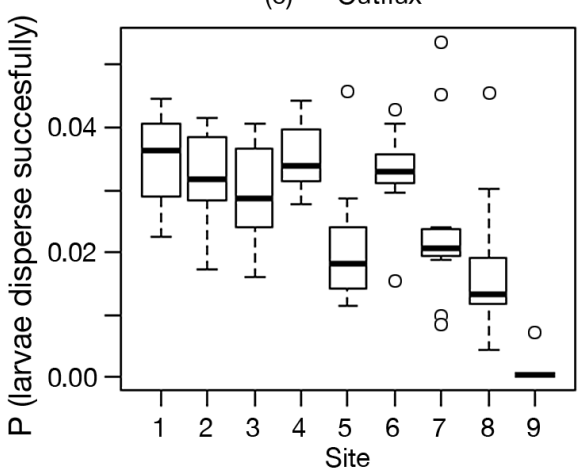

(b) Influx

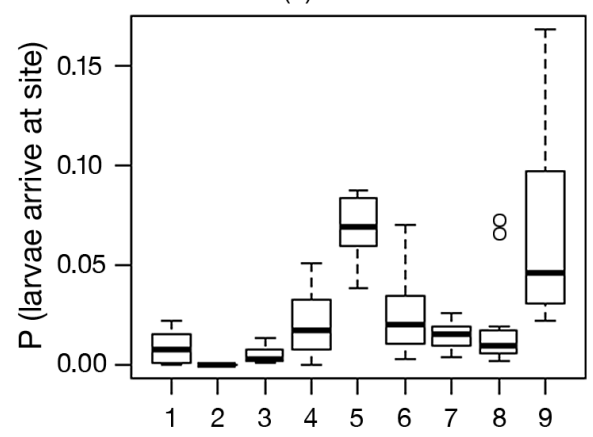

(d) Betweenness

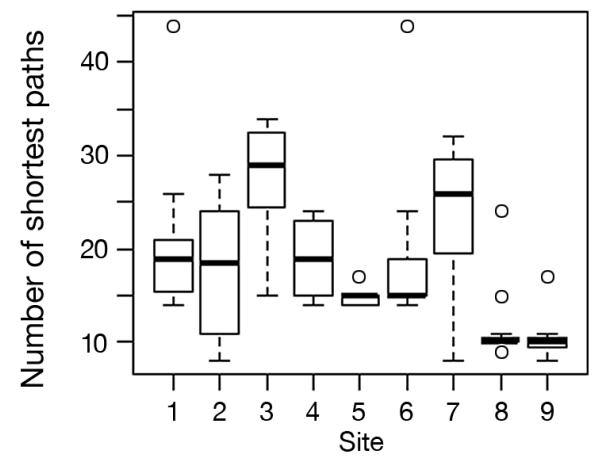

of 4 plankton tows) found non-0 counts of larvae. At only 1 location was there more than 1 non-0 count. The general lack of lice is consistent with the findings of another survey conducted over the same period in nearby Loch Lihnne (Salama et al. 2011). While welcomed by fish farmers, unfortunately this situation means that the data cannot provide a meaningful comparison with model results.

The farm lice counts are more useful. Numbers were generally low during the study period, but lice were found at all sites at some point. Some sites showed a consistent lack of juvenile lice stages, despite the presence of adult lice. We assumed that this arose from misidentification of juveniles as later life history stages, so we used the total lice count in all classes (average number of lice fish $^{-1}$ ) at each farm as our principal metric. Due to variability and data overlap issues, average model connectivity over the 12 separate monthly wind scenarios were compared with total lice counts over the corresponding 12 mo of the production cycle.

Fig. 6 presents the comparison of lice counts with various metrics. A multiple regression was not attempted due to the limited number of data points, and the fact that some model outputs were indirectly obtained from others. The mean current speed was very similar at all sites (Fig. 2a), and no relationship was found between this and lice count. Motivated by the idea that self-infection might be an important factor in allowing site lice populations to multiply rapidly, we also compared the proportion of low current speed records in model output and real observations with lice counts. The relationships here were also weak (Fig. 6a,b), particularly in the case of data current speeds (although the only available records span periods of time that are distinct from the lice data collected). Interestingly, for the sites in the main southern section of the loch (Sites 1-7), the number of fish at each site appears to correlate with the lice count per fish (Fig. 6c). However, those in the narrow northern arm (Sites 8 and 9) experienced much higher numbers.

Sites 8 and 9 receive larvae dispersing from the other sites (due to prevailing wind direction), but do not supply many lice in the opposite direction (Table 1). We therefore compared metrics computed from the time-averaged connectivity matrices with lice counts. Of these, the clearest relationships were observed with site influx (outflux and betweenness did not appear to offer any explanation for observed lice densities). Self-infection probability appears to match well with lice count, though Site 8 still has a high lice count given its level of self-infection (Fig. 6d). This situation does not change in the comparison with total influx from all sites, as predicted by the model (Fig. 6e). Finally, the ratio of self-infection to external infection was compared with lice count in Fig. 6f. Discounting Site 2, sites which experienced 

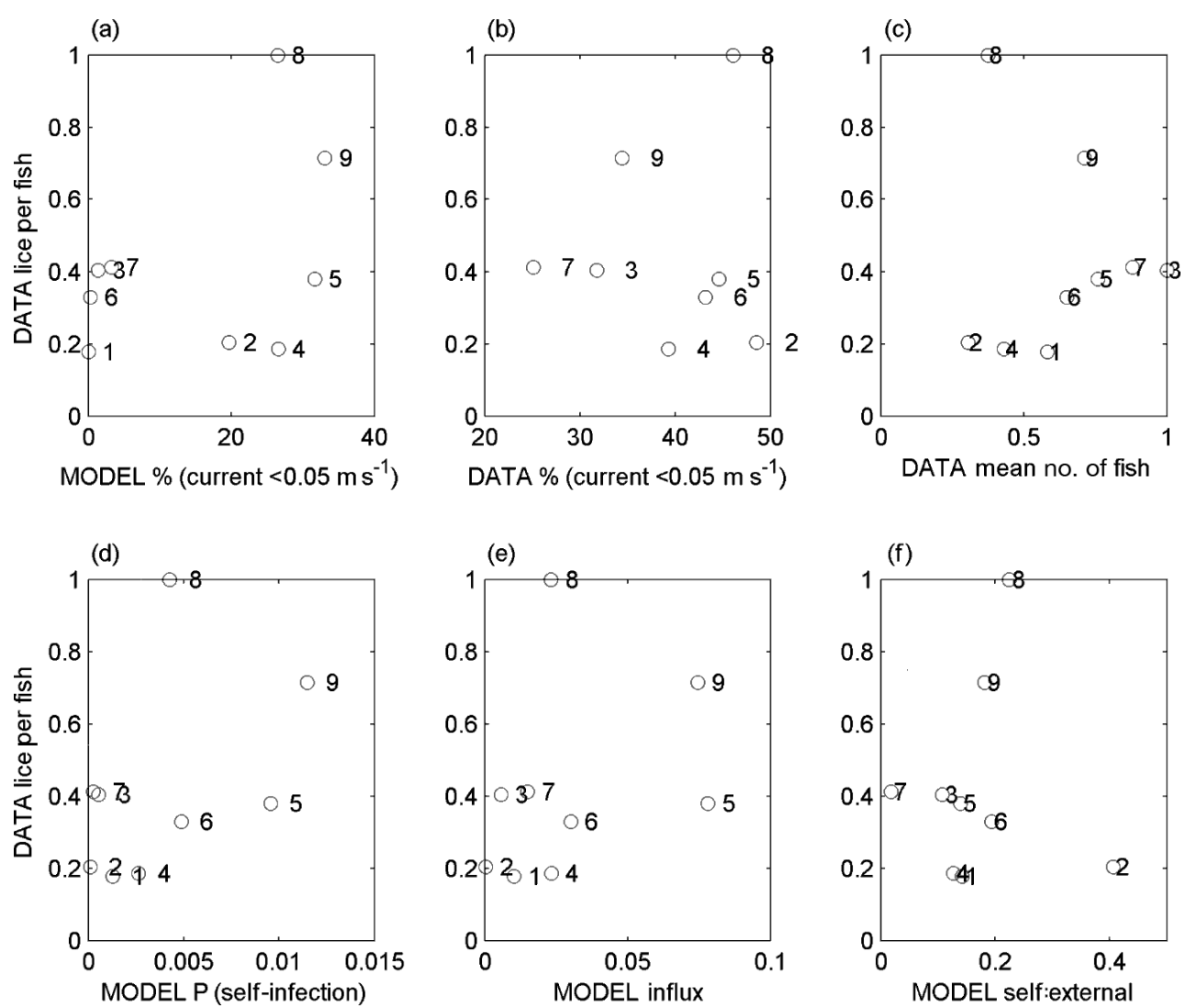

Fig. 6. Lepeophtheirus salmonis. Farm data total lice count per fish (all classes), averaged over the entire year for each site and normalised, versus various metrics: (a) model proportion of current records less than $0.05 \mathrm{~m} \mathrm{~s}^{-1}$, (b) data proportion of current records less than $0.05 \mathrm{~m} \mathrm{~s}^{-1}$, (c) data average number of fish per site (normalised), (d) model probability of self-infection, (e) model average influx, (f) model ratio of self-infection to external infection

higher relative self-infection in the model appeared to have higher lice counts. These discrepancies are considered further in the 'Discussion'.

\section{Ranking site importance}

All sites in the loch are well connected by direct dispersal connections to other sites in the loch, and there are no cut-nodes, which would divide the network into multiple smaller networks if removed.

Of the graph theoretic measures described above, betweenness theoretically provides the best summary of overall site importance to dispersal between sites (influx and outflux are 'one-sided' measures, and do not take into account up- and downstream effects of sites on dispersal). By this measure, site 3 is consistently the most important site for overall connectivity, followed by site 7 . The sites are located centrally amongst the other sites. In this case, it may be that betweenness helps to account for the effect of the sites being central to the network as a whole (leading to accumulation of larvae over time). However, the utility of this idea depends upon whether 'self-infectors' are the primary cause of lice outbreaks, or whether sites with high throughput are more important. The connectivity analysis omits the actual reproductive cycle of lice, and temporally and spatially varying parent populations. We are currently working to include this information into a dynamic model driven by our connectivity predictions.

\section{DISCUSSION}

We have presented an approach that incorporates hydrodynamic and biological modelling with graph/ network analysis in order to gain insight into the spread of sea lice between salmon aquaculture sites. Similar modelling studies are underway in many salmon producing areas, with all main producing countries represented, although these have generally stopped short of computing graph metrics, or 
comparing results with site lice abundances. Some recent work in Norway has also produced favourable results (L. Asplin pers. comm.), and the results here suggest that the explicit consideration of site connectivity measures is an avenue worth following.

Average annual site influx (measured by summing incoming connection probabilities) was the best predictor of observed site lice abundance over a salmon production cycle. Three sites experienced higher lice numbers relative to other sites than predicted by the model. Interestingly, 2 of these sites had very high betweenness in the network, a metric suggestive of high importance to overall connectivity, and this might explain the high observed lice counts. Using these metrics directly to measure site importance may be unwise, as they implicitly assume that the number of larvae produced at each site is identical. Consequently, measured importance of pathways between pairs of sites may be more or less than it is in reality. The importance of sites to the sea lice metapopulation will be easier to determine by dynamic spatio-temporal modelling of lice populations, under a variety of initial infection scenarios. Connectivity matrices can be applied directly here to understand how realistic variation in dispersal between sites is likely to impact lice populations at each site and in the loch in general.

Various factors prevented the application of model results at finer temporal scales; some of these factors related to the available data. In the case of the plankton data, almost no sea lice larvae were found, which prevented any meaningful comparison. In the case of the farm site lice data, the salmon production cycle in Loch Fyne was coming to a close over the first 4 mo during which realistic connectivity scenarios could be generated. More overlap might have allowed month-by-month comparison of observed lice counts with model predictions. However, there was also some possible misclassification of lice stages in the data: some sites had consistent presence of adult lice, but not juveniles. It is possible that juvenile lice escaped detection, or arrived and developed into later stages between sampling events, but this is not a plausible explanation in all cases. This meant that combining values from all lice stages was necessary, and that month-by-month comparison becomes inappropriate (as lice in later stages have been present for several weeks by the time of sampling).

It is thus perhaps slightly unreasonable to expect a perfect match between the model and the data. Mismatches come in 2 forms: predicted presence of lice (high influx) when none are observed, or predicted lack of lice when relatively large numbers are observed. The former is difficult to explain without con- sidering the possibility that the hydrodynamic model circulation pattern is not correct, but was not observed in this study. The latter was observed here (Sites 8 and 9 in all panels of Fig. 6) and could also be caused by erroneous hydrodynamic circulation. At Site 3 (very low predicted self-infection or overall influx, but moderate lice counts), observations showed a much higher proportion of very low current speeds than the model output, and so self-infection may be higher in reality than the predicted value (the model predicted a lower proportion of very low current speed observations than were observed for all sites, but this was an extreme case). There were other notable issues with hydrodynamic predictions, such as incorrect residual flow direction at 2 sites ( 2 and 7). At Site 2, this was not accompanied by a mismatch between model influx and observed lice count, but at Site 7, it was. Furthermore, if these sites were high producers of lice larvae, and there were many sites in their vicinity, this could have an impact on the ability to predict lice abundance over time. Ascertaining the accuracy of these predictions would thus be a priority for further work. Hydrodynamics at Site 8 appeared to be predicted relatively well by the model, but there are other possible explanations.

Firstly, the underestimate of lice numbers at these sites could be due to additional infection of the sites in question from other sources, such as wild fish. Argyll Fisheries Trust (2012) found that of the rivers in Loch Fyne, those in the upper section of the loch were the ones supporting the largest populations of wild salmon. In the lower loch, the only river in which juvenile salmon were found was very close to Site 7 . It seems very likely that wild fish may have passed close to the farm sites, particularly those in the upper loch, which is much narrower and must be negotiated en route to the upper loch river catchments (Sites 8 and 9). The high lice counts noted at these 2 sites might thus be explained in this way. A second possibility is that subtleties in the true (multi-generational, spatio-temporal) population dynamics are not captured by these simple metrics based on a single dispersal cycle. Indeed, comparing average metrics with the average density ignores any temporal patterns in lice numbers that occur, and their impacts on the system. Dynamic modelling based upon the same connectivity matrices may offer insights here. In the present model, the rate of self-infection relative to external infection varied dramatically between sites (from around 2 to $68 \%$ over the entire year; average values given in Table 1), and it may be expected that this will alter the accumulation of lice at particular sites over time. Sites that have high levels of self- 
infection are likely to exhibit positive feedback via the reproductive cycle. It is also likely that sites with low actual current velocities will experience reduced dispersion of lice larvae; larvae produced at sites with low current velocities and/or dispersion will not be spread thinly around the loch.

In summary, we found that a combined modelling approach enabled us to gain insight into the patterns of how lice spread between aquaculture sites within a confined sea loch. By incorporating realistic variation in environmental and seasonal forcing, average population densities at sites in the metapopulation could be partly explained. The connectivity matrix provides a useful way to understand the spread of larvae and may assist in making management decisions relating to sites. However, improved fidelity is likely to be gained by the application of dynamic models to understand the temporal dynamics of lice populations, and this work is currently in progress.

Acknowledgements. This project was funded by a grant from the European Fisheries Fund (EU), number MI-C-30037. We thank 4 anonymous reviewers for their comments, which greatly improved the manuscript.

\section{LITERATURE CITED}

Aldrin M, Lyngstad TM, Kristoffersen AB, Storvik B, Borgan O, Jansen PA (2011) Modelling the spread of infectious salmon anaemia among salmon farms based on seaway distances between farms and genetic relationships between infectious salmon anaemia virus isolates. J R Soc Interface 8:1346-1356

Amundrud TL, Murray AG (2009) Modelling sea lice dispersion under varying environmental forcing in a Scottish sea loch. J Fish Dis 32:27-44

Argyll Fisheries Trust (2012) Loch Fyne Rivers Project: summary of 2011 fish populations, habitat surveys and potential habitat management initiatives. Argyll Fisheries Trust, Inveraray, Argyll

Askeland Johnsen I (2011) Dispersion and abundance of salmon lice (Lepeophtheirus salmonis L.) in a Norwegian fjord system. MSc thesis, University of Bergen

Asplin L, Boxaspen K, Sandvik AD (2004) Modelled distribution of salmon lice in a Norwegian fjord. ICES CM 2004/P:11. International Council for the Exploration of the Sea, Copenhagen

Ayata S, Lazure P, Thiebaut E (2010) How does the connectivity between populations mediate range limits of marine invertebrates? A case study of larval dispersal between the Bay of Biscay and the English Channel (North-East Atlantic). Prog Oceanogr 87:18-36

Bailey RJE, Birkett MA, Ingvarsdóttir A, Mordue (Luntz) AJ and others (2006) The role of semiochemicals in host location and non-host avoidance by salmon louse (Lepeophtheirus salmonis) copepodids. Can J Fish Aquat Sci 63:448-456

Bjørn PA, Sivertsgård R, Finstad B, Nilsen R, Serra-Llinares
RM, Kristoffersen R (2011) Area protection may reduce salmon louse infection risk to wild salmonids. Aquacult Environ Interact 1:233-244

Burridge L, Weis JS, Cabello F, Pizarro J, Bostick K (2010) Chemical use in salmon aquaculture: a review of current practices and possible environmental effects. Aquaculture 306:7-23

Chen C, Beardsley RC, Cowles G (2006) An unstructured grid, finite-volume coastal ocean model: FVCOM user manual. MEDM, University of Massachusetts-Dartmouth, North Dartmouth, MA

Code of Good Practice Management Group (2011) A code of good practice for Scottish finfish aquaculture. Scottish Salmon Producers' Organisation. www.thecodeofgoodpractice.co.uk

Costello MJ (2006) Ecology of sea lice parasitic on farmed and wild fish. Trends Parasitol 22:475-483

Costelloe M, Costelloe J, O'Donohoe G, Coghlan NJ, Oonk M, Van der Heijden Y (1998) Planktonic distribution of sea lice larvae, Lepeophtheirus salmonis, in Killary Harbour, west coast of Ireland. J Mar Biol Assoc UK 78: 853-874

Edwards A, Sharples F (1986) Scottish sea lochs: a catalogue. Scottish Marine Biological Association, Oban

> Fox CJ, Mccloghrie P, Young EF, Nash RDM (2006) The importance of individual behaviour for successful settlement of juvenile plaice (Pleuronectes platessa L.): a modelling and field study in the eastern Irish sea. Fish Oceanogr 15:301-313

> Fox CJ, McCloghrie P, Nash RDM (2009) Potential transport of plaice eggs and larvae between two apparently selfcontained populations in the Irish Sea. Estuar Coast Shelf Sci 81:381-389

Gilbert CS, Gentleman WC, Johnson CL, DiBacco C, Pringle JM, Chen C (2010) Modelling dispersal of sea scallop (Placopecten magellanicus) larvae on Georges Bank: the influence of depth-distribution, planktonic duration and spawning seasonality. Prog Oceanogr 87:37-48

Gillibrand PA (2002) Observations and model simulations of water circulation and transport in Loch Fyne, a Scottish fjord. FRS Marine Laboratory, Aberdeen

Heuch PA, Karlsen E (1997) Detection of infrasonic water oscillations by copepodids of Lepeophtheirus salmonis (Copepoda Caligida). J Plankton Res 19:735-747

> Heuch PA, Bjørn PA, Finstad B, Holst JC, Asplin L, Nilsen F (2005) A review of the Norwegian 'National action plan against salmon lice on salmonids': the effect on wild salmonids. Aquaculture 246:79-92

Heuch PA, Gettinby G, Revie CW (2011) Counting sea lice on Atlantic salmon farms-empirical and theoretical observations. Aquaculture 320:149-153

Knights AM, Crowe TP, Burnell G (2006) Mechanisms of larval transport: vertical distribution of bivalve larvae varies with tidal conditions. Mar Ecol Prog Ser 326:167-174

Krkošek M, Bateman A, Proboszcz S, Orr C (2010) Dynamics of outbreak and control of salmon lice on two salmon farms in the Broughton Archipelago, British Columbia. Aquacult Environ Interact 1:137-146

Lees F, Baillie M, Gettinby G, Revie CW (2008) The efficacy of emamectin benzoate against infestations of Lepeophtheirus salmonis on farmed Atlantic salmon (Salmo salar L.) in Scotland, 2002-2006. PLoS ONE 3:e1549

> McLean PH, Smith GW, Wilson MJ (1990) Residence time of the sea louse, Lepeophtheirus salmonis K., on Atlantic salmon, Salmo salar L., after immersion in fresh water. 
J Fish Biol 37:311-314

Morton A, Routledge R (2005) Mortality rates for juvenile Pink Oncorhynchus gorbuscha and Chum O. keta salmon infested with sea lice Lepeophtheirus salmonis in the Broughton Archipelago. Alsk Fish Res Bull 11: 146-152

Murray AG, Gillibrand PA (2006) Modelling salmon lice dispersal in Loch Torridon, Scotland. Mar Pollut Bull 53: 128-135

North EW, Schlag Z, Hood RR, Li M, Zhong L, Gross T, Kennedy VS (2008) Vertical swimming behavior influences the dispersal of simulated oyster larvae in a coupled particle-tracking and hydrodynamic model of Chesapeake Bay. Mar Ecol Prog Ser 359:99-115

Salama NKG, Murray AG, Rabe B (2011) Modelling dispersal of salmon lice in a large fjordic system: Loch Linnhe, Scotland. MODSIM2011, 19th International Congress on Modelling and Simulation, 12-16 Dec 2011, Perth, p 2156-2162

Stien A, Bjørn PA, Heuch PA, Elston DA (2005) Population dynamics of salmon lice Lepeophtheirus salmonis on Atlantic salmon and sea trout. Mar Ecol Prog Ser 290: 263-275

Editorial responsibility: Bengt Finstad, Trondheim, Norway
Stucchi DJ, Guo M, Foreman MGG, Czajko P, Galbraith M, Mackas DL, Gillibrand PA (2010) Modeling sea lice production and concentrations in the Broughton Archipelago, British Columbia. In: Jones S, Beamish R (eds) Salmon lice: an integrated approach to understanding parasite abundance and distribution. Wiley/Blackwell, Oxford, p 117-150

Sundelöf A, Jonsson PR (2011) Larval dispersal and vertical migration behaviour-a simulation study for short dispersal times. Mar Ecol 33:1-11

Treml EA, Halpin PN, Urban DL, Pratson LF (2008) Modeling population connectivity by ocean currents, a graphtheoretic approach for marine conservation. Landscape Ecol 23:19-36

Turrell WR (1990) Simulation of advection and diffusion of released treatments in Scottish sea lochs. DAFS Marine Laboratory, Aberdeen

Werkman M, Green DM, Murray AG, Turnbull JF (2011) The effectiveness of fallowing strategies in disease control in salmon aquaculture assessed with an SIS model. Prev Vet Med 98:64-73

Zijl F (2009) Development of the Firth of Clyde 2008 flood forecasting model. Deltares, Delft

Submitted: August 29, 2012; Accepted: November 15, 2012 Proofs received from author(s): December 10, 2012 